\title{
Comment on "Correlation between Dynamic Heterogeneity and Medium-Range Order in Two-Dimensional Glass-Forming Liquids"
}

\author{
François Sausset $*$ and Gilles Tarjust \\ Laboratoire de Physique Théorique de la Matière Condensée, Université Pierre et Marie Curie - Paris 6, \\ UMR CNRS 7600, 4 place Jussieu, 75252 Paris Cedex 05, France
}

In a recent letter, Kawasaki et al. [1] study glass formation in a 2-dimensional $(2 D)$ model of polydisperse repulsive disks. They give numerical evidence for a direct relation between slowing down of the relaxation, extension of some medium-range order, and development of dynamic heterogeneities. We disagree with the authors' interpretation on two points which we believe to be potentially important for understanding the glass transition.

First, contrary to what suggested in [1], the order that develops with increasing density when polydispersity is large enough for thwarting crystallization $(\Delta \gtrsim 9 \%)$ should not be contrasted with icosahedral order in $3 D$ (metallic) liquids and glasses and, for that reason, is not appropriately characterized as "crystalline". The local order detected by Kawasaki et al. is hexatic/hexagonal, which represents the locally preferred order of the liquid, just like icosahedral order in $3 D$. When polydispersity is larger than a threshold value $\Delta_{T}$, this liquid local order cannot extend over the whole sample. This frustration is akin to the geometric frustration that prevents tiling of $3 D$ Euclidean space by icosahedral order [2, 3]. The parallel between the two situations is sketched in Fig. 1. In the presence of frustration, crystalline order is either absent or different from the liquid locally preferred order.

Secondly, the observations reported in [1] nicely fit
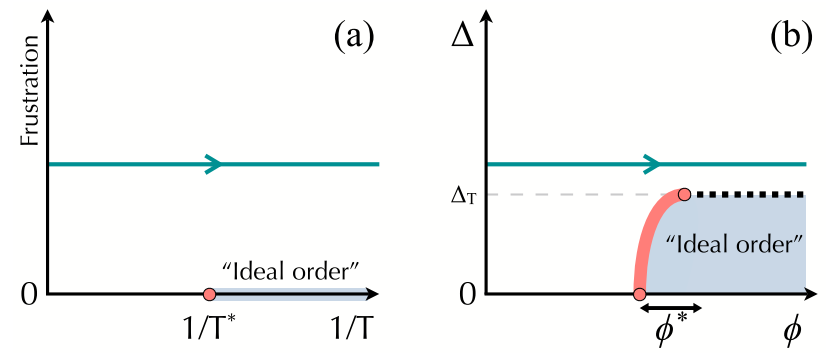

FIG. 1: Schematic diagrams: (a) frustration/temperature for a $3 D$ system; (b) polydispersity/density for a $2 D$ polydisperse system. In both cases the (critical or weakly first order) transition to a phase with long-range extension of the locally preferred structure of the liquid ("ideal order") is avoided in the range of interest for glass formation: in nonzero frustration in (a) and for $\Delta$ larger than $\Delta_{T}$ in (b) (full straight line with arrow). In (a) the transition occurs at a single $T^{*}$ whereas in (b) a continuous transition occurs for a range of density (shown by the arrow around $\phi^{*}$ ) with the transition becoming strongly first order (and irrelevant) in the almost horizontal part shown by a short-dashed line. (The phase diagram of (b) is adapted from [4, [5].) in the frustration-limited domain (FLD) approach [2, $\underline{3}$ ] based on the above frustration description: for large enough polydispersity (i.e. in the presence of frustration), the continuous or weakly first-order transition occuring in the narrow range between $\phi_{I}(\Delta=0)$ and $\phi_{I}\left(\Delta_{T}\right)$ is avoided (see Fig. 1) and marks the onset of cooperative slow dynamics and dynamic heterogeneity, both being associated with the "frustration-limited domains" (clearly visible in Fig.2a of [1]). As stressed in [2, 3], the more frustrated (here, the larger the polydispersity), the less collective the behavior, hence the less "fragile" the liquid, as indeed observed in [1]. Within the FLD approach there is no need to invoke an unreachable ideal glass transition point $\phi_{0}$ to explain the phenomenology. (This turns out to be relevant in the case of $2 D$ polydisperse systems of repulsive disks, for which evidence has been given that there is no such ideal transition [6].) Our interpretation is rather that the avoided singularity leads, beyond some crossover $\phi^{*}$, to a scaling behavior according to which the relaxation time $\tau_{\alpha}$ and the size $L^{*}$ of the FLD's with extended locally preferred liquid order (here hexatic/hexagonal order) behave as

$$
\log \left(\frac{\tau_{\alpha}}{\tau_{0}}\right) \sim L^{* \theta}, L^{*} \sim B\left(\frac{\phi-\phi^{*}}{\phi^{*}}\right)^{x}+\text { constant }
$$

with $B$ decreasing with increasing frustration and $\theta, x$ two exponents whose value depends on the space dimensionality. In the notations of [1], $L^{*}$ identifies with $\xi$ (hence with $\xi_{6}$ ) and, apparently, $\theta \simeq 1$. We checked that the data of Fig.4a of [1] can indeed be fitted by the above formula with $x \simeq 3, \phi^{*} \simeq \phi_{I}$, and $B$ monotonically decreasing as polydispersity increases. This description therefore emphasizes the role of the avoided transition (in zero or very small polydispersity) in triggering the slowing down of relaxation.

* Electronic address: sausset@lptmc.jussieu.fr

† Electronic address: tarjus@lptl.jussieu.fr

[1] T. Kawasaki, T. Araki, and H. Tanaka, Phys. Rev. Lett. 99, 215701 (2007).

[2] G. Tarjus, S. A. Kivelson, Z. Nussinov, and P. Viot, J. Phys.: Condens. Matter 50, R1143 (2005).

[3] D. Kivelson, S. A. Kivelson, X. Zhao, Z. Nussinov, and G. Tarjus, Physica A 219, 27 (1995).

[4] M. R. Sadr-Lahijany, P. Ray, and H. E. Stanley, Phys. Rev. Lett. 79, 3206 (1997). 
[5] S. Pronk and D. Frenkel, Phys. Rev. E 69, 066123 (2004).

[6] L. Santen and W. Krauth, Nature 405, 550 (2000). 